\title{
An Efficient Way to Detect and Recognize the Overlapped Coins using Otsu's Algorithm based on Hough Transform Technique
}

\author{
Deepika Mehta \\ Assistant Professor \\ Computer Science and Engg. \\ DAVIET, Jalandhar \\ Punjab
}

\author{
Anil Sagar \\ Assistant Professor \\ Information Technology \\ BCET, Gurdaspur \\ Punjab
}

\begin{abstract}
The competency to detect and to recognize the different denomination of coins is an essential expertise in the human beings life. This paper represents different algorithms for the detection and the recognition of coins which are highly overlapped. The proposed system includes three principal steps detection, extraction and recognition. This system is efficient in detecting and recognizing the coins from both the sides. The system uses Otsu's algorithm for segmentation process, for detecting overlapping uses hough transform and recognizing the coin uses radius thresholding.
\end{abstract}

\section{General Terms}

Segmentation, Coin Detection, Coin Recognition.

\section{Keywords}

Otsu's Algorithm, Hough Transform, Coin Detection, Coin Recognition.

\section{INTRODUCTION}

Before we begin to discuss about the coin detection and its recognition when they are overlapped, its significant to understand the efficient use of coins. The coins which have been acting as the daily need of any human beings. Counting of coins is very difficult process in today's life of humans. So in spite of their daily use in their lives its also becoming an important task for the researchers to work on their detection and recognition of coins in order to reduce the load of human to count, detect and to recognize the coins or when the coins are overlapped. Detection and recognition of coins are the important principals before the overlapping of coins are to be highlighted. Various algorithms and the approaches used in the detection and recognition of coins. The various approaches used were hough transform, Artificial Neural Networks, Heuristic Approach, Registration technique, Vector Quantization, Histogram Modeling used to detect and recognize the coin image. Detection and recognition of coin was done based on the different parameters such as radius, thickness, size, shape. The various types of coin recognition methods are available in the day today life such as mechanical based method, electromagnetic method, Image processing methods based on the different parameters. The images which were recognized and detected from these system were easily faked out not with accurate results. In spite of such problems arising various methods, algorithms and techniques were introduced to detect and recognize the coins.

\section{PREVIOUS WORK}

In 2003[8] M.Nolle et.all described the pattern recognition methods in order to detect and recognize the large volume of coins. They performed experiments by taking coins from around 30 countries which were easily separated and recognized. It shows $96 \%$ of known coins classified and $99 \%$ unknown coins rejected. Further work can extended on to the improvement of recognition. In 2009[7] used the heuristic approach and tried to achieve $97 \%$ accuracy in recognition of coins. It used Hough transform algorithm in order to detect and recognize the coins on the basis of various parameters. In 2011[5] the recognition of the coin was done by considering both sides of the coin image using Artificial Neural Networks. It shows $97.74 \%$ correct recognition was achieved with $2.26 \%$ miss-recognized. In 2012[1] the recognition was done using Circular Hough transform. The coins of the different denomination were used. Various images of coin were used to evaluate the performance of the image. It shows $94 \%$ of recognition of the different denomination of coins.

The proposed work includes different algorithms such as Otsu's Algorithm for segmentation, Hough Transform for detection when the image is overlapped and recognition of coin image using Radius Thresholding. All of the algorithms are used for the pre-processing, detection and recognition of an image when overlapped. In section 3 we have proposed system and its implementation are given. Then section 4 the results are provided. Then in section 5 we have concluded the whole work.

\section{PROPOSED SYSTEM}

The image used in the set-up includes a computer and camera. The camera used having the resolution of 300 X 400 pixels. The images used in the system can be resized according to the need of the users. The image taken from far tends to be small and if image taken from near tends to be large so for this user defined values are taken in order to minimize this problem. However the proposed system is the detection and recognition of coins using various algorithms and operation. The detection and recognition process can be divided into the various steps. The architecture for the detection and recognition of coins is shown in fig 1 . 


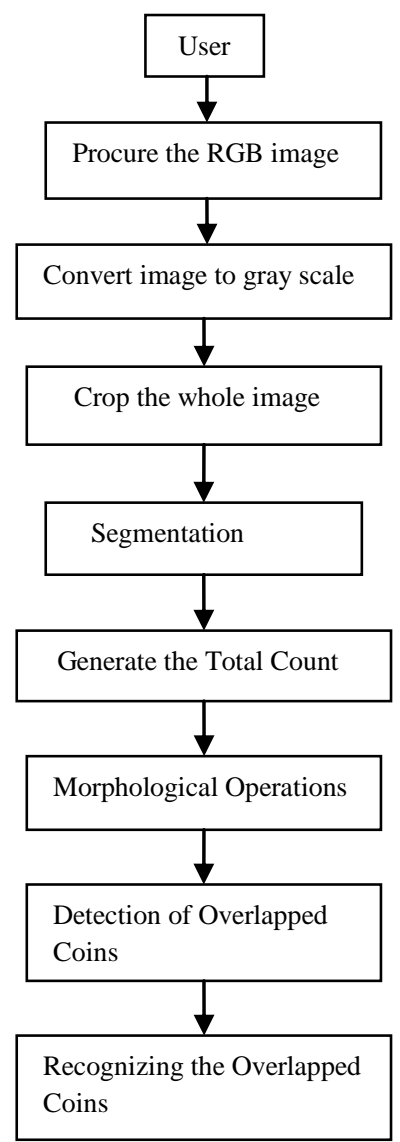

Fig. 1: Architecture for Detection and Recognition of overlapped coins

\subsection{Procure the RGB Image of the Coin}

The initialization is done by the user in order to obtain an image from the computer or the camera used. Once the image is selected the RGB image of coin is scanned from the both sides.

\subsection{Convert the Colored (RGB) image to}

\section{Grayscale}

As the RGB image chosen is converted to the grayscale. If the image first chosen is already grey so no requirement to convert it. If the size of the image is small then RGB image can be utilized for detection and recognition and if image size large so it is required to convert it.

\subsection{Cropping of an Coin Image}

Once both steps are completed it is required to crop the image in order to have the exact image of coin.

\subsection{Segmentation and Deriving Total Count}

Once the cropping of an image is done the next step is to divide the image into various segments depends on the total number of the coins present in an image. This is done with the help of the algorithm known as Otsu's Algorithm. This algorithm is a histogram based thresholding method and known as bwlabel command. Whenever any image is analyzed it is first segmented then the corresponding thresholding method is done over it. In order to attain the overlapping in an image Morphing operations can be used.
Morphed images are those which modify an image smoothly by defining some values. Fig. 2 shows a sample image, Fig.3 shows the segmentation of sample image and Fig. 4 shows the total count.

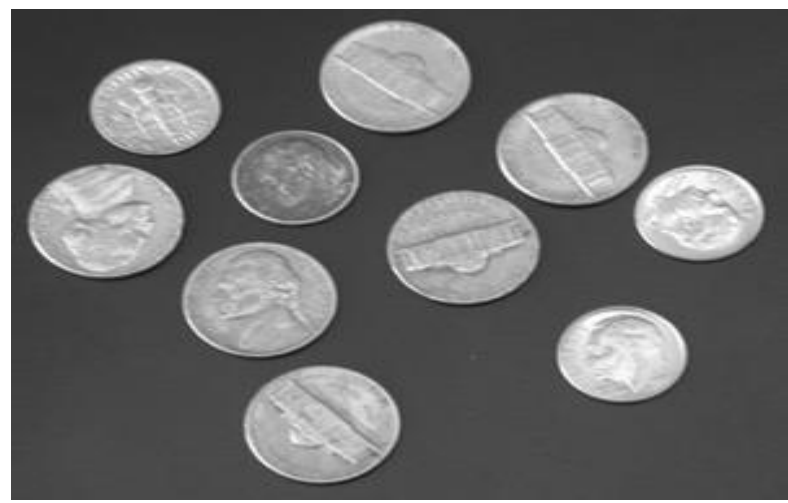

Fig 2: Sample Image for Segmentation and Total Count
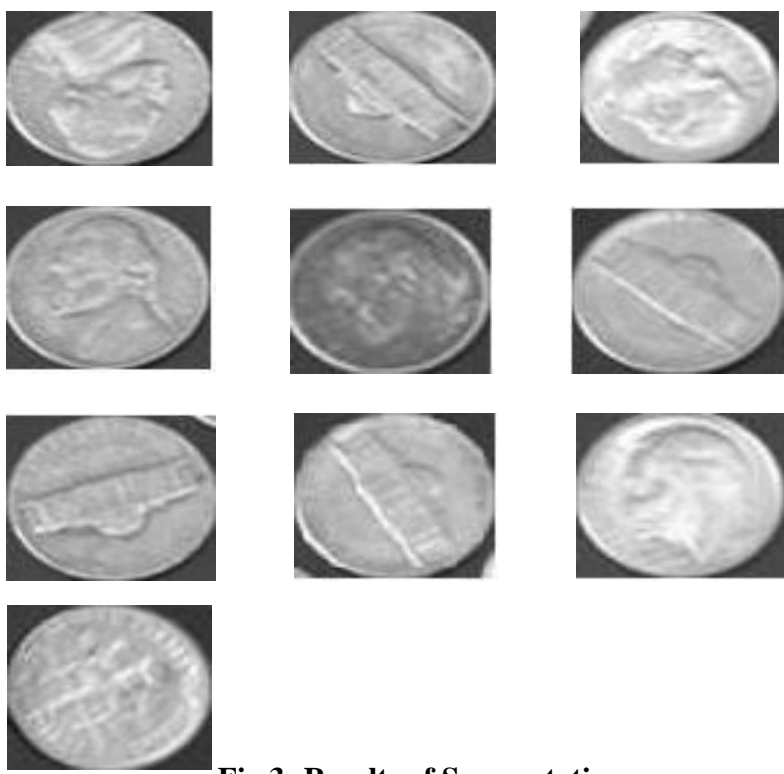

Fig 3: Results of Segmentation

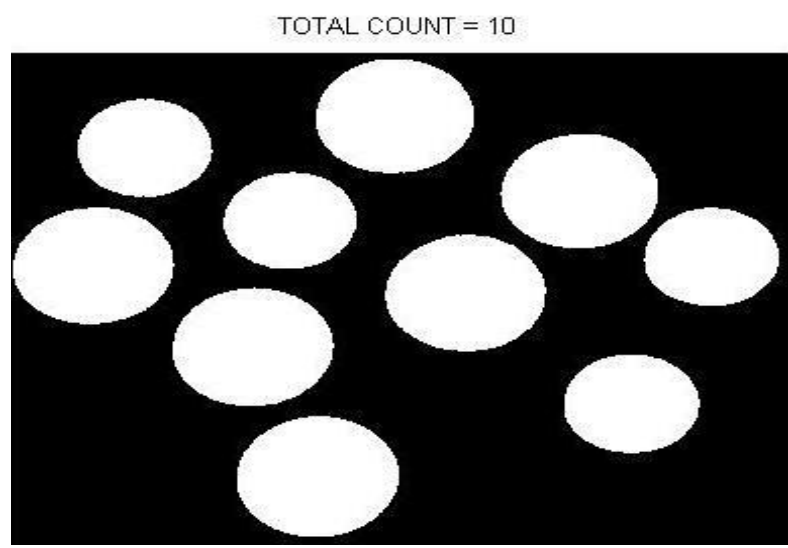

Fig 4: Results of Total Count

\subsection{Morphological Operations}

Once segmentation is complete, morphological operations can be used to remove imperfections in the segmented image. Morphological Operations are used to enhance the image contrast, detection of edges, segmented into various regions. These operations are performed for the overlapped images. 
Once the operation is performed on the image, after the removal of imperfection arise during the segmentation either it erodes an image or it enlarges the two objects used in the sample image. This depends upon the morphological value provided by the user for an overlapped image. Fig. 5 and 6 shows the sample image and the result of this operation.

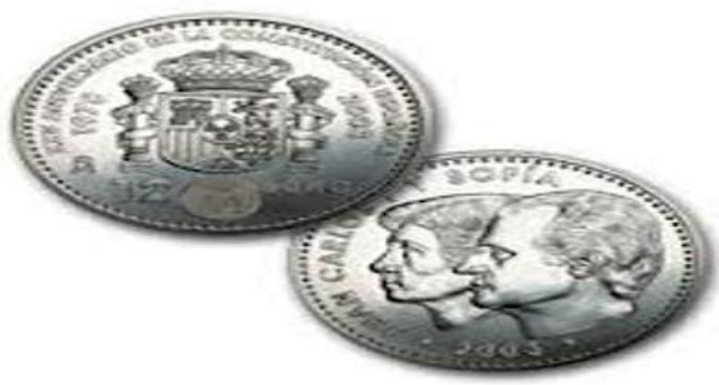

Fig 5: Sample Image used for Morphological Operations

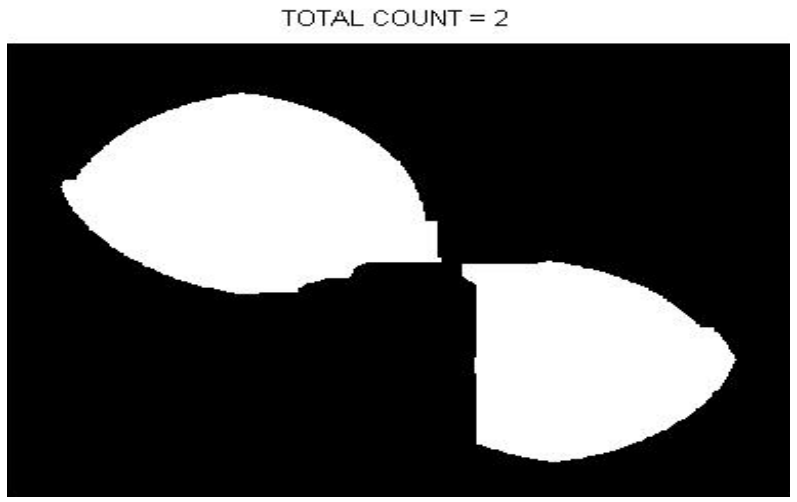

Fig 6: Result of the Morphological Operations

\subsection{Detection of the Overlapped Coins in an Image}

Once the total count and segmentation is completed the next step is the use of the morphing operations on the images. These operations are used to remove the imperfections. Once morphing operations are used then the next is to identify the overlapped coins in an image. This can be achieved using the technique known as Hough Transform. The coins can be identified by taking parameter as radius which thus can be user defined radius values. Hough Transform is a feature extraction technique used in image analysis. There are many techniques and methods used to detect the coins but not the detection of the overlapped coins up to radius level. Our proposed study solves this problem by detecting the overlapped coins up to the radius level, the value defined by the user. The main advantage of using this technique is the high reliability in the detection of overlapped coins in an image. Fig. 7 and 8 shows the detection of overlapped coins.

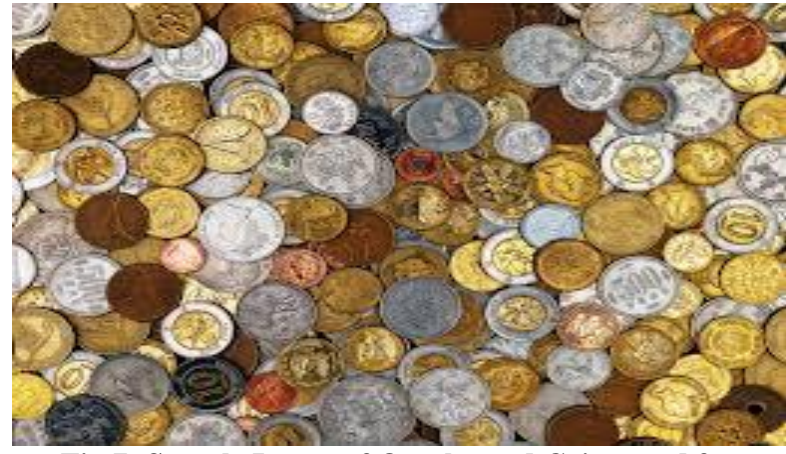

Fig 7: Sample Image of Overlapped Coins used for Detection

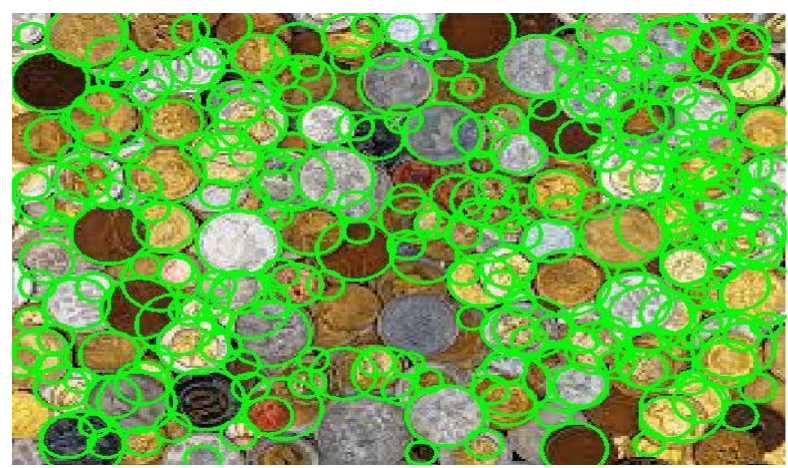

Fig 8: Result of Detection in an overlapped Image

\subsection{Recognition of the Coin Image}

Once the image with overlapped coins are detected, the next step is to recognize it. Recognition is a difficult process in the view of computers and when it comes to an overlapped coins in an image it becomes more difficult. Results of recognition were achieved for overlapped and non overlapped coins in an image but were not satisfying for overlapped coins in an image as the accuracy was $40 \%$ to $50 \%$ shown in Fig 9 . Detection and recognition of an image is a great challenge. Detection is achieved with satisfied results. Here recognition is done by Radius Thresholding. The radius which thus affects the size of the edges in an image whether the image is small or large where thus thresholding helps in controlling the brightness of an image and sharpens the image. Lower values of the edges are to be sharpened rather than values which are high. Finally coin image are recognized with their total count in an image. Fig 9, Fig 10 and 11 shows the recognition of coins in an image.

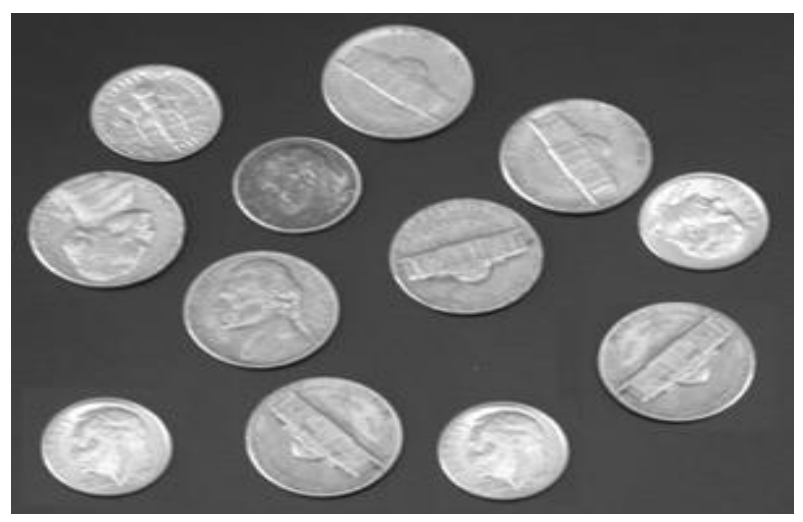

Fig 9: Sample Image for Recognition 


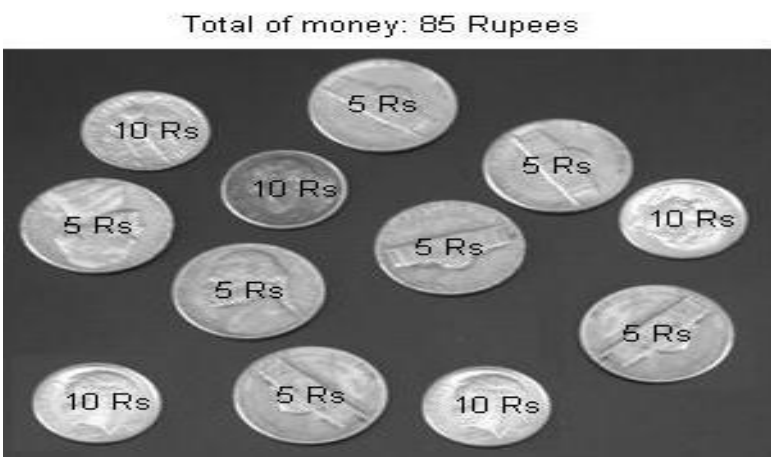

Fig 10: Result of Recognition

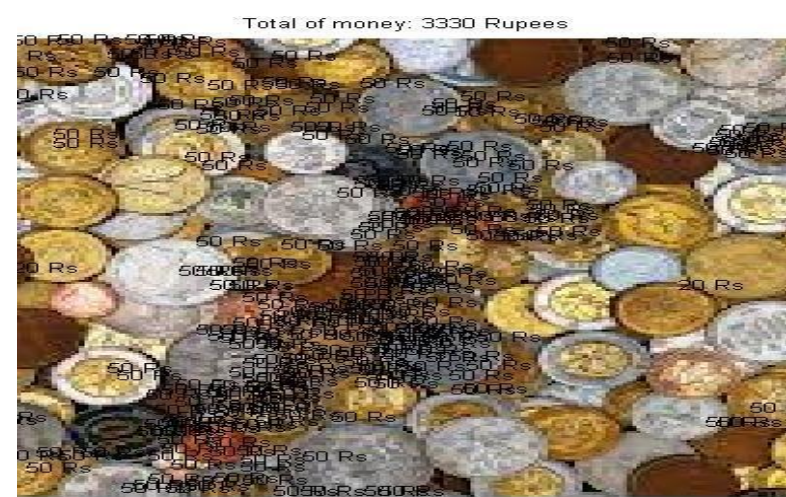

Fig 11: Result of Recognition of an Overlapped Coin

\section{RESULTS}

Various samples of coin images were pre - processed by different techniques and algorithm. The sampled images can be resized as when required by the users. This resizing of the image helps in fast processing of images. The runtime of the Otsu's algorithm for detecting and recognizing overlapped coins in an image was from 10 seconds to 20 seconds. The performance of the proposed system was accurate for many situations. The algorithm was used to obtain the thresholding value. The Hough transform was used to detect the circular objects in the images. The algorithm was insensitive for the images used. The images had undergone all the stages in detecting and recognizing the coins. The accuracy achieved in detecting the overlapped coins in an image was $91.11 \%$. To obtain the experimental results of the detection of the coins overlapped up to the radius level can be described by equation as

$$
\text { Percentage }=\frac{\text { detected coins }}{\text { total number of coins }} \times 100
$$

\section{CONCLUSIONS}

The proposed study was to detect and recognize the overlapped coins using the Otsu's algorithm based on the hough transform technique. The algorithm used to provide with the optimal threshold value for detection. By using the algorithm and techniques the results are evaluated. The proposed system can be used in any of the machines that are based on the coin detection and recognition.

The scope of the proposed system was to detect and recognize the overlapped coins, which was achieved. The detection of the overlapped coins in an image provided with the satisfying results but recognition results were not satisfied when the coins were overlapped with each other.
The main advantages of the system were less computation time and inexpensiveness.

Further the research can be extended in improving the results of recognition of the several coins that are overlapped with each other.

\section{REFERENCES}

[1] Jain, N., and Jain, N. 2012 Coin Recognition Using Circular Hough Transform, International Journal of Electronics Communication and Computer Technology, Vol 2, Issue 3, 2249-7838

[2] Liangwongsan S., Marungsri B., Oonsivilai R. and Oonsivilai A. 2011 Extracted Circle Hough Transform and Circle Defect Detection Algorithm, World Academy of Science, Engineering and Technology.

[3] Reisert M., Ronneberger O. and Burkhardt H. A Fast and Reliable Coin Recognition System. University of Freiburg, Computer Science Department, 79110 Freiburg i.Br., Germany.

[4] Pendse M. and Wang Y. Automated Coin Detection on Android Phone.

[5] Bawa S., and Modi S. 2011 Automated Coin Recognition System using ANN, International Journal of Computer Applications, Vol 26, Issue 4, 0975-8887.

[6] Rizon M., Yazid H., Saad P., Shakaff A.Y.M., Saad A.R., Sugisaka M., Yaacob S., Mamat M.R., and Karthigayan M 2005 Object Detection Using Circular Hough Transform, American Journal of Applied Sciences 2 (12):1606-1609, ISSN 1546-9239.

[7] Velu C.M. and Vivekanandan P. 2009 Indian Coin Recognition System of Image Segmentation by Heuristic Approach and Hough Transform, International Journal Open Problems Compt.. Math., Vol 2, Issue 2.

[8] Nolle M., Penz H., Rubik M., Mayer K., Hollander I., and Grance R. 2003 Dagobert - A New Coin Recognition and Sorting System, proceeding in VIIth Digital Image Computing:Techniques and Applications, Sydney.

[9] Xiao Z. and Weij P. 2006 Detection of Circle based on Hough Transform, Transducer and Micro System Technologies, Vol 8 pp: 25-34.

[10] Bremananth R., Balaji B., Sankari M. and Chitra A. 2005 A New Approach to Coin Recognition using Neural Pattern Analysis, proceedings of IEEE Indicon conference, pages 366-370.

[11] Tresanchez M., Palleja T., Teixido M. and Palacin J. 2009 Using the Optical Mouse Sensor as a Two-Euro Counterfeit Coin Detector, Sensors, 7083-7096.

[12] McNeill S., Schipper J., Sellers T. and Nechyba M.C. Coin Recognition using Vector Quantization and Histogram Modelling. Machine Intelligence Laboratory, University of Florida at Gainesville, FL 32611. 